\title{
Utilizzo del modello Calpuff per la valutazione della qualità dell' aria
}

\author{
Annamaira Shara Ferruzzi ${ }^{1}$ and Davide Rizzo ${ }^{1}$ \\ ${ }^{1}$ Affiliation not available
}

\begin{abstract}
La legislazione europea e il suo recepimento nella normativa nazionale incoraggiano lo sviluppo e l'uso di sistemi modellistici di qualità dell'aria che sono considerati di primaria importanza nelle valutazioni preliminari di qualità e utili per completare il contenuto informativo delle misure dirette. Il funzionamento dei modelli di dispersione consiste nel simulare gli effetti di una o più sorgenti di emissione in termini di concentrazione degli inquinanti emessi in corrispondenza di fissati recettori. Il presente articolo analizza il sistema modellistico Calpuff applicato ad un caso studio sull'impianto di processo di un'azienda chimica di Ferrara per valutarne il comportamento.
\end{abstract}

\section{Introduzione al comparto e ai modelli di dispersione}

Il comparto atmosferico è uno dei comparti più importanti nell'ambito della valutazione di impatto ambientale in quanto sono numerose i fenomeni, sia antropici che naturali, che possono andare ad alterare sia direttamente che indirettamente il suo stato. E' necessario definire in maniera più precisa alcuni concetti di base, come il concetto di inquinate, di inquinamento e di qualità dell'aria. Nel DPR 203/1988 si può definire col termine inquinamento dell'aria:

ogni modificazione della normale composizione o stato fisico dell'aria atmosferica, dovuta alla presenza nella stessa di una o più sostanze in quantità e con caratteristiche 
tali da alterare le normali condizioni ambientali e di salubrità dell'aria; da costituire pericolo ovvero pregiudizio diretto o indiretto per la salute dell'uomo; da compromettere le attività ricreative e gli altri usi legittimi dell'ambiente; alterare le risorse biologiche e gli ecosistemi ed i beni pubblici e privati

La normativa di riferimento nazionale è il Decreto Legislativo 152/2006 che nella parte V definisce e caratterizza i limiti delle emissioni. Successivamente il Decreto Legislativo 13 agosto 2010, n.155 recepisce la direttiva 2008/50/CE introducendo anche i valori limite di qualità dell' aria alle immissioni. Ai fini della valutazione di impatto ambientale risultano ancora utili le prescrizioni data dal Decreto Ministeriale 28/12/88 in cui vengono definiti gli elementi minimi per la caratterizzazione del comparto. Nel primo allegato del DM sono descritte le componenti: ogni comparto si divide in componenti e ogni componente può avere uno o più fattori ambientali. Nel secondo allegato invece va a descrivere gli elementi minimi, ovvero gli indicatori, da dover descrivere con riferimento alle componenti ed ai fattori ambientali del comparto specifico.

Dal DM28/12/88 possiamo individuare gli elementi necessari per un'analisi sufficiente del comparto:

- dati meteorologici convenzionali (temperatura , precipitazioni, umidità relativa, vento ecc.)

- dati di concentrazione di specie gassose e di materiale particolato

- caratterizzazione dello stato fisico dell'atmosfera

- caratterizzazione preventiva dello stato di qualità dell'aria (gas e materiale particolato)

- localizzazione e caratterizzazione delle fonti inquinanti

- previsione degli effetti del trasporto (orizzontale e verticale) degli effluenti mediante modelli di diffusione di atmosfera

- previsioni degli effetti delle trasformazioni fisico-chimiche degli effluenti

Si capisce quindi che caratterizzare tale comparto significa andare a studiare non solo la qualità dell'aria ma anche quelle che sono i fenomeni di tipo meteoclimatiche che regolano il trasporto 
delle sostanze emesse. Quest'ultimo aspetto richiede pertanto la modellazione matematica di fenomeni molto complessi in cui la turbolenza atmosferica e le trasformazioni foto-chimiche giocano un ruolo fondamentale.

In particolare è necessario andare a caratterizzare il comportamento di quello strato di atmosfera a diretto contatto col suolo caratterizzato da connotazioni chimico-fisiche che lo rendono di fatto uno strato di confinamento entro cui si racchiude il fenomeno della dispersione atmosferica. Sulla base delle caratteristiche di emissione e dei fattori meteorologici, un modello di dispersione può essere utilizzato per prevedere le concentrazione nello spazio di una sostanza emessa da una sorgente. I modelli di dispersione possono avere come base teorica un differente approccio nel considerare lo spostamento delle particelle nello spazio: E' possibile adottare per lo studio di queste un approccio Euleriano che fissa un sistema di riferimento entro cui la posizione della particella viene individuata oppure un approccio Lagrangiano in cui si segue la particella nel suo movimento. Entrambi gli approcci non sono integralmente adottabili senza apportare delle semplificazioni al sistema di equazioni differenziali che governano il fenomeno fluidodinamico per via delle difficoltà nel caratterizzare lo sviluppo della turbolenza. Per rendere trattabile il problema si è optato per un'integrazione numerica su un numero ridotto di equazioni differenziali e approssimare le rimanenti. Un contributo notevole alla modellazione proviene dalla Teoria della similarità che tramite la processione di una enorme quantità di dati ricavati dalla registrazione delle condizioni atmosferiche nel tempo è riuscita a dare una forma semi-empirica ai fenomeni fisici dello strato atmosferico più superficiale e ricavare quindi relazioni di validità generale che mettono il profilo verticale delle principali variabili di interesse in funzione della quota e dei parametri della turbolenza. Attraverso queste è stato possibile creare dei modelli più semplici e pratici a patto di fare i conti con forti limitazioni dovute alle iniziali ipotesi semplificative. I limiti sono sono un applicabilità solo in situazioni di forte omogeneità superficiale (zone pianeggianti), omogeneità di copertura e un'evoluzione dei fenomeni turbolenti quasi stazionario. Un ulteriore passo per affrontare la mo- 
dellazione del fenomeno è descrivere la forma con cui un'emissione si disperde in atmosfera. In genere le forme più semplici sono il puff, concepito come singolo sbuffo di gas emesso, e il plume, il classico pennacchio delle ciminiere. Nel tempo è stato osservato un comportamento ricorrente di queste forme di emissioni nelle diverse condizioni atmosferiche. Una delle semplificazioni più importanti è quella che adottano i cosiddetti modelli stazionari, cioè considerare che i fenomeni atmosferici (e quindi turbolenti) si mantengano costanti e inalterati in un intervallo di tempo tale da rendere rappresentativa la stima del valor medio della concentrazione. Uno dei modelli più famosi tra i tanti prodotti grazie a questa semplificazione, nonché uno dei più utilizzati per i fini ingegneristici dove non è richiesta una conoscenza molto precisa della dispersione atmosferica, è il modello stazionario Gaussiano, che fa una serie di ipotesi semplificative molto limitanti, tra cui le più incisive sono la superficie piana e la stazionarietà dei fenomeni atmosferici

\section{Casto Studio}

Si è analizzato uno studio dove è stato utilizzato il software Calpuff applicato alle emissioni dell'azienda LyondellBasell di Ferrara che opera nel settore chimico. Le sostanze analizzate emesse dal camino dell'impianto analizzato non sono cancerogene per l'uomo ma presentano caratteristiche di tossicità e per alcune sono definiti limiti alle emissioni (D.Lgs 152/06). Il modello Gaussiano a puff utilizzato dal software si basa sull'ipotesi che qualsiasi emissione di inquinante da parte di una sorgente puntuale può essere vista come l'emissione in successione di una sequenza di piccoli sbuffi di gas ciascuno indipendente dall'altro. Tali porzioni di fumo, una volta emesse, evolvono indipendentemente nello spazio e nel tempo in base alle caratteristiche di spinta acquisite all'emissione e in base alle condizioni meteorologiche medie e in base alla turbolenza che incontrano nel loro cammino. Inoltre è un modello di tipo stazionario ovvero considera la turbolenza atmosferica "congelata" durante il suo passaggio. Questa semplificazione permette una facile implementazione del modello ma ne limita l'utilizzo solo a specifiche condizioni che ne limitano il campo di applicabilità. Non è infatti possibile: 
- tenere conto dell'orografia del territorio

- considerare eventuali trasformazioni chimiche

- tenere conto della deposizione secca o umida delle sostanze emesse

Gli ultimi due effetti, anche se presenti, possono essere trascurati in quanto la loro assenza ci portano ad una sovrastima delle concentrazioni, mentre il primo non presenta problemi trovandosi il caso studio in un territorio pianeggiante. Bisogna prima di tutto definire il dominio di calcolo su cui verrà posta una griglia i cui punti sono definiti secondo le coordinate UTMW-GS84. Le informazioni geofisiche necessarie per la simulazione sono l'andamento del terreno con la quota e l'uso del suolo. Queste informazioni sono da fornire per ogni cella del dominio di calcolo.

Si hanno a disposizione i dati proveniente da una centralina meteo che si trova nei pressi dell'impianto. A questo scopo si è creato un programma che utilizza i dati della centralina per ottenere come output un file che contiene i dati di velocità e direzione del vento, altezza delle nubi, copertura del cielo, temperatura, umidità relativa, pressione e tipologia di precipitazione. Per descrivere l'andamento di velocità media in funzione della quota si è utilizzato una legge esponenziale basata sulle classi di stabilità atmosferiche Pasquill e del tipo di territorio (rurale o urbano). Sono stati poi inserti il profilo di pressione e il profilo di temperatura. Per ciascuna sostanza emessa dal camino dell'impianto di Ferrara bisogna inserire i valori relativi alle proprietà delle sostanze emesse (diffusività, costante di Henry, ecc.). Bisogna poi fornire i dati della sorgente (altezza, temperatura emissione, diametro ecc.) e definire la posizione dei ricettori dove analizzare l'andamento temporale delle concentrazioni. I recettori sono stati disposti ad altezza d'uomo (1.5m) per ottenere l'effettiva concentrazione con cui la popolazione entra in contatto e infittiti in corrispondenza dell'impianto. Il modello consente di implementare nell'area di studio l'effetto del Il building downash cioè il disturbo causato da edifici, o da altre costruzioni che agiscono da ostacolo, sulla dispersione delle sostanze in aria. In generale un ostacolo crea delle turbolenze indotte dalla forza 
del vento che agisce su di esso, si ha quindi una modifica sulla naturale traiettoria del vento. Può succedere che la turbolenza locale richiama il pennacchio verso il basso e di conseguenza sottovento all'ostacolo si può avere un aumento di concentrazione di inquinanti. Degli ostacoli inseriti, sono stati automaticamente esclusi quegli edifici ritenuti dal software ininfluenti, analizzando solo quelli intorno al camino in grado di produrre un maggior effetto considerando la loro posizione e dimensione. Avviata la simulazione si può visualizzare l'andamento spaziale delle concentrazioni e di tracciare le isoplete, cioè le curve che uniscono i punti a uguale concentrazione. Dal confronto della concentrazione simulata con le concentrazioni limite imposte a livello legislativo per la tutela della salute, è possibile valutare il rischio da esposizione prolungata. Per quanto riguarda le sostanze emesse dal camino in esame, esse non presentano particolari caratteristiche di pericolosità per la salute umana, e il decreto 155/2010 non impone limiti alle immissioni. Essendo le sostanze emesse non contemplate dal D.lgs $155 / 10$, si è fatto riferimento alle reference concentrations (RfC) proposte dall'EPA nel database IRIS. Queste concentrazioni tutelano la salute umana da esposizione continuativa considerando l'intero arco della vita e sono utilizzate negli Stati Uniti per il calcolo del rischio. Tenendo conto delle sostanze emesse che rientrano in tale database è stata analizzata la loro concentrazioni risultando essere dell'ordine dei microgrammi su metro cubo, mentre i limiti di riferimento sono circa tre ordini di grandezza superiori, e si è quindi ben lontani da avere problemi per esposizione a lungo termine. Un altro risultato notevole della simulazione è l'andamento temporale ai singoli ricettori delle concentrazioni medie giornaliere delle sostanze emesse. L'andamento temporale ci permette di ricavare informazioni importanti ad esempio in fase di progetto di un nuovo impianto o di autorizzazione alle emissioni, come i massimi di concentrazione e il numero dei superamenti annuali di fissati valori di concentrazione. Dai dati ottenuti con la simulazione si è ricavato l'andamento spaziale, all'interno della griglia di calcolo, delle medie annue di concentrazione e l'andamento temporale delle concentrazioni in corrispondenza di alcuni recettori di esempio. Questi valori possono essere confrontati con livelli di riferimento per la qualità dell' aria e, dagli andamenti temporali si possono estrarre i massimi o la frequenza di 
superamento di determinate soglie, qualora esse siano definite a livello normativo. Si è poi svolta una seconda simulazione che tiene conto dell'effetto di Building Downwash considerando gli ostacoli alla dispersione delle sostanze in aria. Questo effetto può infatti creare zone con maggiore o minore concentrazione rispetto alla simulazione su terreno piano. Dalla differenza tra le due simulazioni calcolata relativamente al caso senza ostacoli, si hanno differenze anche fino al 10\% (in positivo e in negativo). Se si considerano le medie mensili, queste differenze raggiungono anche il $60 \%$. Questo è spiegato dal fatto le differenze significative si hanno solo sporadicamente, quando si verificano particolari combinazioni di velocità e direzione del vento che accentuano l'effetto di building downwash.

\section{Conclusioni}

Con questo studio è stato analizzato un caso applicativo con lo scopo di fornire una conoscenza più approfondita dei modelli di dispersione tramite l'applicazione di un software. Le basi strutturali su cui queste si poggiano e quindi i limiti che li caratterizzano sono fondamentali per un loro corretto utilizzo sul campo in quanto non sempre le condizioni in cui ci si trova ad applicarli rispettano i limiti con cui tali modelli semplificati sono stati concepiti. Ai fini della caratterizzazione del comparto atmosferico si evidenzia l'utilità di questi strumenti che possono essere utilizzati anche come base di partenza per il calcolo del rischio per la salute umana o per studi eco-tossicologici e di migrazione delle sostanze emesse.

\section{References}

1.Negri, E. Utilizzo del modello Calpuff per la valutazione della qualità dell'aria da emissioni . (Università di Bologna, 2011).

2.LyondellBasell Ferrara. 
3.ARPA FVG - Agenzia Regionale per la Protezione dell'Ambiente del Friuli Venezia Giulia. 
Figure Captions

Figure 1. Foto di Marc Wieland

Figure 2. Area oggetto di simulazione

Figure 3. Air quality 


\section{Figures}

"begin-figure"“"begin-center"“includegraphics[width=0.70"columnwidth]-figures/marc-wieland-

zrj-TPjcRLA-unsplash/marc-wieland-zrj-TPjcRLA-unsplash"“caption-FotondiøMarcøWieland-“label-100274”

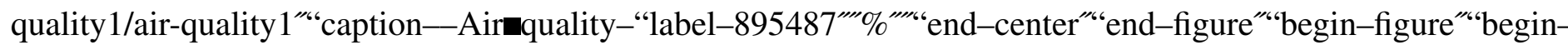
unrau-47679-unsplash/sebastian-unrau-47679-unsplash"“"end-center"“"end-figure" 